\title{
Technology supporting infection prevention and control training in Africa
}

\author{
Gerard Lacey* $^{* \ddagger}$, Buyiswa Lizzie Sithole ${ }^{\dagger}$ and Anna Vorndran ${ }^{\dagger}$ \\ *Electronic Engineering, Maynooth University, Co Kildare, Ireland Email: gerard.lacey@mu.ie \\ †Infection Control Africa Network (ICAN), Cape Town, South Africa Email: anna@icanetwork.co.za, lizzie@icanetwork.co.za \\ ${ }^{\ddagger}$ SureWash, Dublin 2, Ireland Web: www.surewash.com
}

\begin{abstract}
Infection Control Network Africa (ICAN) is the largest infection prevention and control (IPC) organisation in Africa. It provides IPC education programs and actively supports IPC implementation to reduce healthcare-associated infections (HAIs), such as COVID-19, Ebola, etc. Good quality clinical hand hygiene is the foundation of IPC. Still, it is challenging to teach as it involves a new psychomotor skill and develops new hand hygiene habits appropriate to the clinical setting. In 2019 ICAN Train-The-Trainer (TTT) program began using the SureWash technology platform to support both in-person education and the cascade of training to other staff in the facility. This paper describes the need, the technology platform, program rollout before and during the COVID-19 outbreak, the impact and the lessons learned. We conclude by providing some observations on the future use of technology for infection control training.
\end{abstract}

Index Terms-Infection Control, education, Mixed Reality Simulator, Mobile App

\section{INTRODUCTION}

Good hand hygiene is a cornerstone of patient safety. The World Health Organisation (WHO) has a comprehensive set of guidelines on hand hygiene [1]; these cover the "When" of hand hygiene i.e. the 5 moments and the "How" of hand hygiene i.e. the "WHO 6-step technique". The benefits of training healthcare workers (HCW) in hand hygiene behaviours is well established. Studies have shown a 50\% improvement in asepsis following training [2] and corresponding reductions in hospital-acquired infections (HAIs)[3].

Infection Control Africa Network (ICAN)[4] was founded in 2008 to facilitate infection control programs, reduce healthcare infections and promote antimicrobial stewardship through education. ICAN's vision is an African continent where safe patient care is ensured by implementing strong evidence-based infection control programs. ICAN is the training partner for the WHO and CDC in IPC training and guideline development in Africa. Some typical short courses offered by ICAN include:

- IPC for Healthcare workers

- IPC for Healthcare managers

- IPC for non-healthcare workers

- Train-the-Trainer in IPC

- Fundamentals of IPC

- Combating Antimicrobial Resistance through IPC

- Introduction to Water, Sanitation and Hygiene (WASH)

978-1-6654-4925-0/21/\$31.00 (c) 2021 IEEE

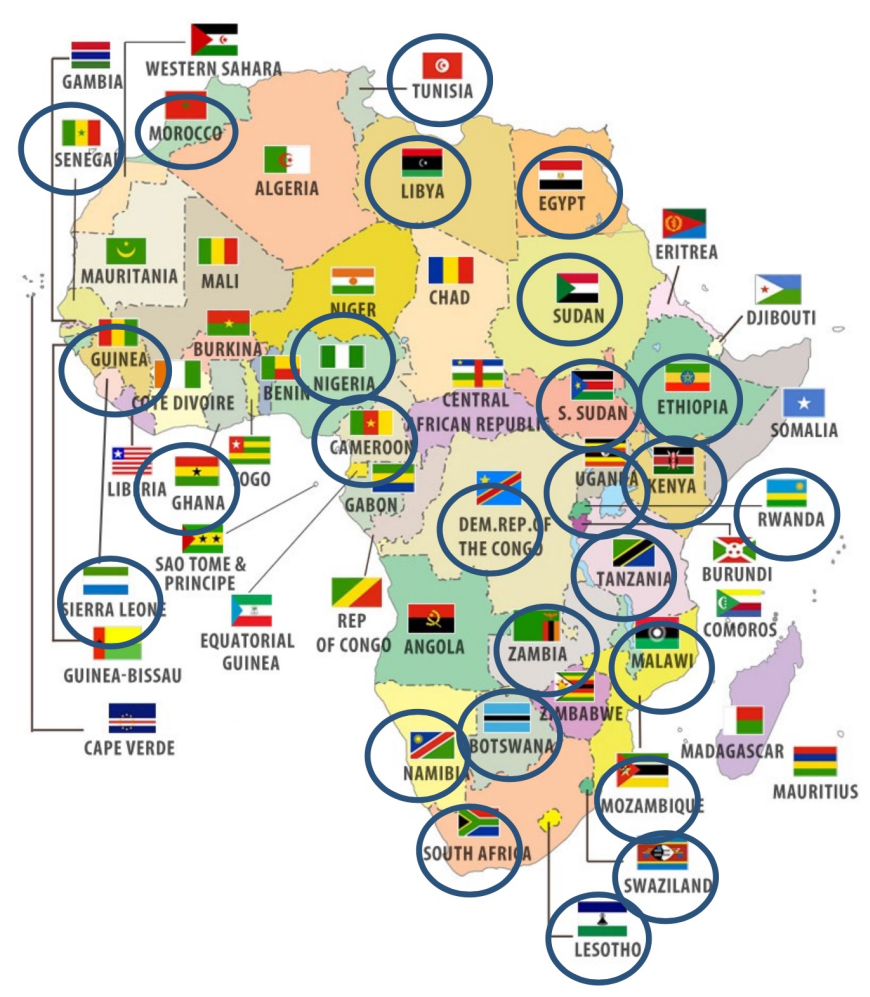

Fig. 1. The circles indicate the ICAN partner counties in Africa

ICAN responded to the Ebola outbreak in Sierra Leone and most recently has provided support to Africa Union, Africa CDC, WHO in dealing with COVID-19. ICAN has been involved since May/June 2020 with "Resolve to Save Lives" [5] in 6 different countries, providing tools, training and assisting facilities in developing their own improvement action plan and evidence-based IPC programs.

This paper describes the use of novel technology to provide hand hygiene training as part of the train-the-trainer courses. We will describe the train the trainer process and the specific needs that required a technology intervention. We will describe the rollout of the program and its impacts. We then discuss the impact of the technology and the lessons learned. Finally, we will outline the future needs of IPC training in Africa. 


\section{TRAIN-THE-TRAINER(TTT) IN IPC}

Many healthcare facilities in Africa are not performing well on hand hygiene and infection control, [6] and Africa consistently performs poorly on the WHO Hand Hygiene Self-Assessment Framework [7]. Low engagement with the WHO 5th May Global Hand Hygiene campaign [8] lead ICAN to develop the "Turn Africa Orange Campaign"[9]. ICAN identified that many facilities struggle to implement hand hygiene programs for their staff and need support in stimulating awareness and building stakeholder engagement for hand hygiene promotion.

Part of ICAN's strategy is to provide Train-the-Trainers (TTT) sessions in regional centres across Africa. This five-day training program is not designed to be totally comprehensive but to introduce basic knowledge of the role of IPC, decontamination, hand hygiene and design of an ideal IPC department. The people are assessed at the end of the course, and those that achieve $80 \%$ and are passionate about IPC are provided with an additional week of adult learning. In this follow on training, they develop their own IPC training material, adapting it to the local culture. ICAN will visit their facilities after 3 months and ensure that the training materials are evidence-based and then examines the Trainer's students. If the students pass, the Trainer is given a certificate of competence. ICAN uses this approach to ensure that evidence-based training is cascaded to the healthcare facilities and that trainer competence is assessed based on their ability to transfer knowledge accurately.

Hand hygiene is the cornerstone of the TTT program, and engagement of participants is good when face-to-face. But it takes time and corrective feedback to develop the "muscle memory" of the WHO 6 poses. As a result, competence in hand hygiene was often very poor. ICAN sought a training tool that would allow Trainers to improve their hand hygiene competence and would help them to cascade competencebased hand hygiene training to their local facilities. It was during this search that ICAN came across SureWash.

\section{The SureWash Platform}

The core of the SureWash platform is a computer vision that automatically measures physical skills and provides realtime feedback to the user on their proficiency[10]. To use the system the user places their hands in the view of the camera. To preserve the user's privacy, the camera faces downwards so that only the hands are shown on the screen, as shown in Figure 2. The SureWash system provides the user with a live image of their hands and provides onscreen feedback about the accuracy of their hand hygiene practice with respect to the WHO hand hygiene poses shown in Figure 3.

The technology has been deployed previously as an Internet of Things(IoT) device over hand hygiene sinks [11], as portable kiosks [12] and mobile phone apps [13]. The portable kiosk version, SureWash GO, is transported between facilities in a rugged travel case and placed on a table or hung on a wall, as shown in Figure 2. The mobile phone app, SureWash Hand Hygiene, works by placing the phone on a horizontal surface such as a table and the user's hand motions are then

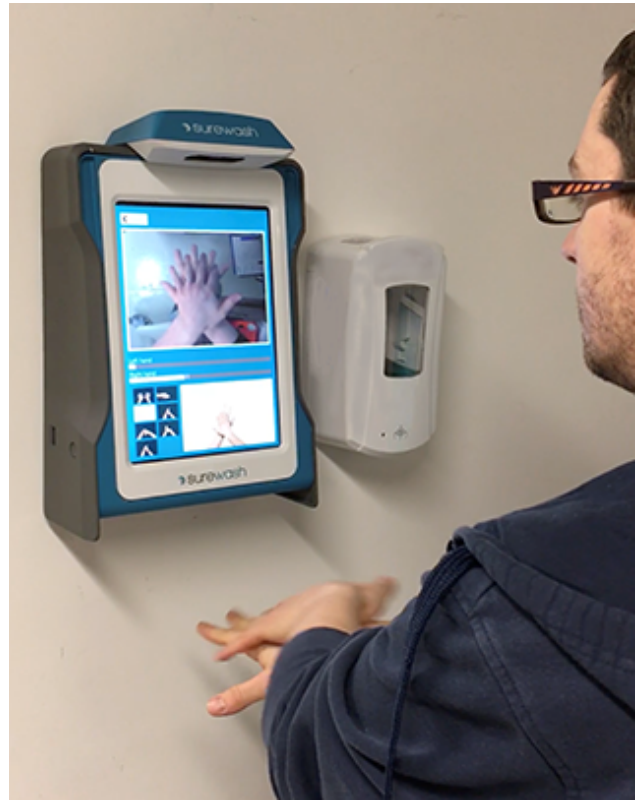

Fig. 2. A SureWash GO unit mounted on a wall. The camera at the top of the unit faces down so as to track the users hands.

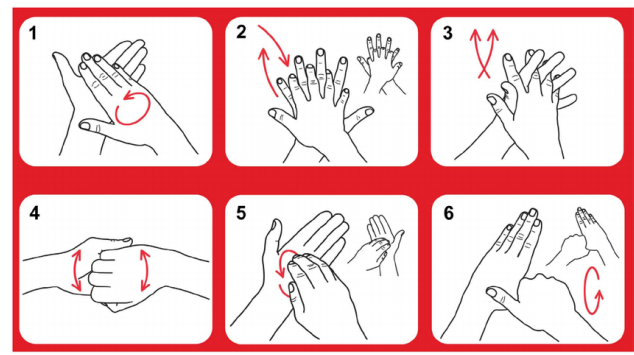

Fig. 3. The 6 poses of the WHO hand hygiene technique

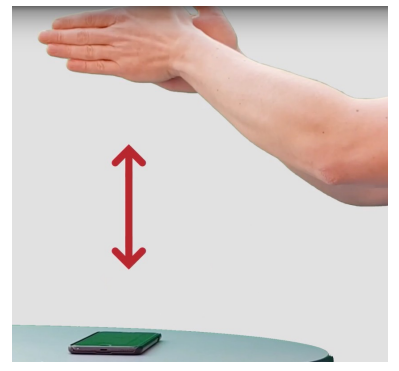

Fig. 4. To use the SureWash Hand Hygiene app, place the phone on a horizontal surface and hand movement is tracked by the front facing camera

tracked by the front-facing "selfie" camera as shown in Figure 4. The app was available free on iOS (iPhone 6 and better) and Android (Samsung 8 and better).

Competence in hand hygiene is built up over multiple practice sessions. As with all psychomotor skills learning, repetition with feedback within a "Deliberate Practice" framework produces the best learning outcomes [14]. Typically between 20 and 30 practice sessions, each lasting about 2 minutes, spread over 2-3 weeks, are required to develop the muscle 
memory of the WHO 6 poses[12]. The learning is divided into 5 layers that scaffold the learning, building up speed and technique until a final assessment where the user completes the WHO technique in order without any prompts. Both the Kiosk and app version of the SureWash system allows users to log in with a user name and password to save their learning progress. The records are available to instructors to monitor engagement and identify any areas of technique that are consistently giving trouble, allowing them to focus their instruction on these areas.

\section{ROLL-OUT OF THE PROGRAM}

The roll-out of the TTT program began in 2018, covering 6 countries: Malawi, Cameroon, Nigeria, Zimbabwe, Uganda and South Africa and has trained staff from 300 facilities. ICAN uses a country targeted approach; a call goes out for people interested in the TTT course, and then the course is delivered in a regional centre. Following the course, the trained staff go back to their facilities and cascade the IPC training to their own staff.

At the beginning of the roll-out, we needed to understand the technology and use it most effectively. We began using the SureWash GO kiosk as a teaching aid for teaching hand hygiene technique, similar to how we used Glo-germ boxes [15]. A Glo-germ box gets staff to cover their hands is a fluorescent gel and then view their hands under a UV light. They are often surprised by the parts of the hands that they miss. The main advantage of SureWash is that it concentrates on developing a good process of hand hygiene that can be repeated every time rather than in a one-off Glo-germ test. The very detailed feedback gives a "light-bulb moment" when staff realise that they are not doing the process correctly. The machine must provide this feedback rather than a person as people are more willing to accept that it is objective. Because of the game-like character of the technology, it does drive a good-natured competition between staff which adds an element of fun to the classes.

In addition to Hand Hygiene, the courses cover basic IPC, costs, risk assessment and hand hygiene promotion. For hand hygiene promotion in developed countries, IPC teams make instructional videos or dance videos to train and motivate staff in clinical hand hygiene. This approach is challenging to adapt in Africa because most of our hospitals don't have the screens to show these videos. For hand hygiene promotion, because most of the staff have smartphones, we encourage the use of the SureWash app as people can use the app at home or at lunchtime with other staff. The scoring mechanism is crucial for generating a sense of competition between staff which helps with hand hygiene promotion. We also encourage patients to use the app as competition between staff and patients can be a good promotional tool.

When preparing for the course, we had informed staff about the app's availability, but it wasn't easy to demonstrate over the phone, and people did not understand the dynamics. However, after the trainees had used the SureWash kiosk, they understood how to use the app and were able to incorporate it into their own teaching and hand hygiene promotion.
Since the pandemic, it has been more difficult to deliver the program. Similar to the rest of the world we use a virtual teaching platform. In the virtual instruction siutation the SureWash app has allowed students to develop good hand hygiene technique even though we cannot be there in person with the SureWash GO kiosk. Once trained the students were able to incorporate the use of the SureWash app into their own teaching.

An example of the impact of the TTT program can be seen in one hospital in Johannesburg. In May 2019 the program was given to the Neonatal Unit(NNU). Hand hygiene training was provided to 10 Consultants, 28 Registrars, 15 Doctors, 1 Cardiologist, 4 Interns, 3 Infection Control Nurses and 20 Nurses. A Hand Hygiene promotion day was held on the 5th of May (World Hand Hygiene Day). Hand Hygiene training was also extended to 30 nursing mothers. Hand hygiene compliance levels at the NNU were monitored before and after training. Compliance with hand hygiene rose from $25 \%$ to $41 \%$. Although it represents a significant improvement, it also indicates that much progress still needs to be made.

\section{LESSONS LEARNED}

During the rollout, we learned how to use the technology successfully by introducing the app after conducting faceto-face or remote training sessions with the Kiosk. We also learned that the app could be a powerful tool to cascade the training. However, two issues had a significant impact on the program's long-term effectiveness: Limitations on the access to data on outcomes and the ability to support virtual teaching.

\section{A. Access to data}

In Africa, there is a lot of bureaucracy and stress relating to the release of data in certain institutions. Facilities are concerned about how the data might be used. Despite the training being approved by the facility owners or ministries, there still needed to be a separate memoranda of understanding (MOU) for data collected during the training period and data on how training cascades within the facility. The issues were how the data was collected, where it would be stored, who has access to that data, and whether it will be kept in their hospital. As a result, it was quite challenging to assess the effectiveness of the training and its impact on the cascade of training to the facility and ultimately on hand hygiene compliance rate and ultimately the impact on the rates of HAIs.

Reliable data is key to an evidence-based approach to improving patient safety. So it would be good to have a multiyear agreement where we could use the data generated by the SureWash kiosks and the apps to understand the facility's ongoing training and then correlate this with the hand hygiene compliance rates and the HAI rates for the facility.

\section{B. Remote teaching tools for IPC}

Since the beginning of the Pandemic, it has been difficult to do face-to-face training. Virtual learning platforms can help provide lecture materials, but it is difficult to assess student engagement during the sessions. For example, with a group of 
20 or 30 participants only two or three respond to instructor questions. Creating "breakout groups" for discussions does help but there is a real problem when demonstrating and assessing physical skills. It would be ideal if we could integrate the SureWash app into a virtual learning platform. We want to be able to have people set their own challenges and quizzes. It would be good to be able to use this "live" within a virtual classroom. Having mini-physical challenges measured live via the app would really help an instructor assess their teaching impact and which aspects of hand hygiene need more attention.

A significant issue that arose during the pandemic was the inappropriate use of Personal Protective Equipment and other basic IPC strategies. The requirement for scenario-based training and assessment became clear. What is required is for students to encounter various situations where they have to perform a quick risk assessment and choose a set of actions that are based on IPC guidelines. This type of scenario-based simulation helps students to transfer their theoretical knowledge into decision making skills relevant to their facilities.

\section{CONCLUSION}

This paper has described the use of a high technology solution for training healthcare workers and patients in clinical hand hygiene training as part of a distributed Train-TheTrainer program in Africa. The technology platform consisted of a mobile kiosk used by the ICAN training staff and a smartphone app that students downloaded onto their personal devices. The app was used to improve their own hand hygiene competence and to help cascade the training within their local facilities. We learned that people would only download the app after having received face-to-face or online instruction. We also learned that the mobile app was a useful tool to help people cascade the training into their own facilities.

While the technology was successful, we also identified that data access restrictions limited the program's impact for reducing Hospital-Acquired infections. We hope that organisations can develop effective data sharing agreements so that programs such as TTT can be even more effective.

The COVID-19 pandemic increased the amount of online training provided. While the SureWash app, in particular, remained useful, we felt that it could be even more effective if integrated with an online learning platform and supported inclass exercises. We also identified the need for scenario-based online training to allow students to perform a risk assessment and select an appropriate IPC strategy.

We have shown in this paper that high technology from developed countries can have an even more significant impact on Hand Hygiene training in Africa due to the deep penetration of smartphones. We also identify that the availability of tools to integrate this technology with live online classes and provide online situation based simulation will further improve the quality and ability to scale the training across the continent.

\section{ACKNOWLEDGMENT}

The authors would like to thank the Support of the Kailo Foundation and SureWash for providing the platform and the supporters and members of the ICAN organisation for supporting IPC education in Africa.

\section{REFERENCES}

[1] WHO et al., WHO guidelines on hand hygiene in health care: first global patient safety challenge clean care is safer care. World Health Organization, 2009.

[2] A. F. Widmer, M. Conzelmann, M. Tomic, R. Frei, and A. M. Stranden, "Introducing alcohol-based hand rub for hand hygiene: the critical need for training," Infect Control Hosp Epidemiol, vol. 28, no. 1, pp. 50-4, 2007. [Online]. Available: https://www.ncbi.nlm.nih.gov/pubmed/ 17230387

[3] B. Allegranzi and D. Pittet, "Role of hand hygiene in healthcareassociated infection prevention," $J$ Hosp Infect, vol. 73, no. 4, pp. 305-15, 2009. [Online]. Available: https://www.ncbi.nlm.nih.gov/ pubmed/19720430

[4] "Infection Control Africa Network ICAN," (Date last accessed 15-June-2021). [Online]. Available: http://www.icanetwork.co.za/

[5] "Resolve To Save Lives," (Date last accessed 15-June-2021). [Online]. Available: https://resolvetosavelives.org/about/press/protecting-hcws

[6] J. Irehovbude and C. A. Okoye, "Hand hygiene compliance: bridging the awareness-practice gap in sub-saharan africa," GMS hygiene and infection control, vol. 15, no. 06, 2020.

[7] C. Kilpatrick, E. Tartari, A. Gayet-Ageron, J. Storr, S. Tomczyk, B. Allegranzi, and D. Pittet, "Global hand hygiene improvement progress: two surveys using the who hand hygiene self-assessment framework," The Journal of hospital infection, vol. 2, no. 100.

[8] A. Peters, T. Borzykowski, E. Tartari, S. Kilpatrick, C.and Mai, B. Allegranzi, and D. Pittet, "'clean care for all-it's in your hands": the may 5th, 2019 world health organization save lives: Clean your hands campaign," Antimicrobial resistance and infection control, vol. 64, no. 8.

[9] “Turn Africa Orange," (Date last accessed 15-June-2021). [Online] Available: https://www.icanetwork.co.za/projects/turn-africa-orange/

[10] A. J. Stewardson, A. Iten, V. Camus, A. Gayet-Ageron, D. Caulfield, G. Lacey, and D. Pittet, "Efficacy of a new educational tool to improve handrubbing technique amongst healthcare workers: A controlled, before-after study," PLOS ONE, vol. 9, no. 9, p. e105866, 2014. [Online]. Available: https://doi.org/10.1371/journal.pone.0105866

[11] G. Lacey, J. Zhou, X. Li, C. Craven, and C. Gush, "The impact of automatic video auditing with real-time feedback on the quality and quantity of handwash events in a hospital setting," American journal of infection control, vol. 48, no. 2, pp. 162-166, 2020.

[12] G. Lacey, M. Showstark, and J. Van Rhee, "Training to proficiency in the WHO hand hygiene technique," Journal of medical education and curricular development, vol. 6, p. 2382120519867681, 2019.

[13] "Surewash Mobile App," (Date last accessed 15-June-2021). [Online]. Available: https://surewash.com/surewash-app-practice-hand-hygiene/

[14] K. A. Ericsson, "Deliberate practice and acquisition of expert performance: a general overview," Acad Emerg Med, vol. 15, no. 11, pp. 988-94, 2008. [Online]. Available: https://www.ncbi.nlm.nih.gov/ pubmed/18778378

[15] "Glo-germ," (Date last accessed 15-June-2021). [Online]. Available: hhttps://www.glogerm.com/ 